J. Austral. Math. Soc. Ser. B 30(1988), 79-88

\title{
A FLEXIBLE STRATEGY FOR WARRING DUOPOLISTS
}

\author{
JOHN RICKARD ${ }^{1}$ AND NEVILLE HATHAWAY ${ }^{1}$
}

(Received October 2, 1986; revised April 27, 1987)

\begin{abstract}
The behaviour of duopolists is considered within a framework that allows for flexibility of the adopted strategy against the rival. In a difficult external climate, a firm may concentrate on its own profit, whereas in a more favourable external climate, it may adopt a more aggressive attitude towards the rival. The strategy considered in this paper permits this flexible approach. The market functions are kept general to allow the widest interpretation of the results.
\end{abstract}

\section{Introduction}

Duopoly and oligopoly problems have received extensive study by economists since the work of Cournot [2]. The reason for this is probably that most industries in the Western Economies can be approximated by either a duopoly or an oligopoly model.

A major deficiency of duopoly and oligopoly models is that they assume a fixed behaviour of the competitors. This is clearly unrealistic. In a difficult sales period, a firm may concentrate solely on its own immediate profit. In a morefavourable climate, it may decide it can afford to wage a war on its rivals, in an attempt to secure a superior position in the industry. If this attempt fails, the firm may revert to a more conciliatory behaviour towards its rivals. A model of a duopoly or an oligopoly thus needs to have this flexibility of strategy built into it.

A model which has some of this flexibility was used by Bishop [1] in the analysis of a duopoly model. He examined the case of duopolists, with constant and equal average costs, producing a homogeneous product subject to a linear

\footnotetext{
${ }^{1}$ The Graduate School of Management, University of Melbourne, Parkville, Victoria 3052, Australia.

(C) Copyright Australian Mathematical Society 1988, Serial-fee code 0334-2700/88
} 
demand. The duopolists are quantity setters, simultaneously setting quantities which are then sold at the appropriate price determined from the demand function. No verbal communication between rivals is allowed and no side payments occur.

Bishop proposed that each duopolist have a reaction schedule to the other's output. This reaction schedule gives a competitor's output as a function of the rival's output. It has three branches. Along the first, or conciliatory branch, a duopolist attempts to maximise the combined profit $\pi_{1}+\pi_{2}$, where $\pi_{i}$ is the profit of the $i$ th duopolist. Consequently, as his rival increases his output, the duopolist must decrease his own output. On the second, or antagonistic branch, a competitor more or less matches his rival's increased outputs with his own increase in output. A competitor tries to ensure that, even if he suffers in the process, the opposition gets no more than what the competitor thinks his rival's share should be. The third, or limiting branch, involves a point beyond which a competitor will not increase his warfare output. He may feel that to go beyond this point places too high a cost upon himself. In transferring from the antagonistic branch to the limiting branch, a competitor's strategy changes from placing direct pressure on his rival to minimising his own losses, whilst not conceding to his rival's demands.

The eventual outcome of the warfare cannot be rationally deduced. It may depend on the tempers of the two competitors-two stubborn competitors may adopt a permanent warfare stance against each other. The duration of the warfare could also depend on the competitors' willingness and ability to sustain recurrent losses. Even if he is neither able nor willing to sustain such losses, a competitor may wish to convey to his rival the opposite impression. Thus an element of "bluffing" is introduced. Bishop comments that the resistance of a competitor to his rival's demands could stem from the suspicion that the rival is bluffing. As bluffing is not easily discernible, this may also give rise to a stalemate.

The existence of a long-term stalemate raises the question of the stability of such solutions. Intuitively one feels that a system with two aggressive opponents would be quite unstable. We shall be concerned with this question in this paper. It will be shown that, with suitable limits on the aggression of each competitor, stable equilibrium solutions occur in the warfare state. Hence long-term stalemates are a distinct possibility.

Finally, it should be noted that we shall not be considering the general question of entry and exit. We are however, considering a possible warfare strategy to be used by existing competitors. A consideration of the problem of exit and entry may be found in, for instance, Friedman [3]. 


\section{Demand, cost and profit functions}

Rather than introduce explicit functions to describe the demand and cost functions pertaining to the market, as in Hathaway et al. [4], we shall use general unspecified functions throughout. Some assumptions about the properties of these functions are necessary in an attempt to make them reflect realistic market behaviour.

Let $x_{i}(t)$ be the output of competitor $i$ at time $t$. The total industry output is then given by $x_{T}(t)=\sum_{i=1}^{n} x_{i}(t), n$ being the number of firms.

The market demand function, $p$, is assumed to be a function of $x_{T}$. The specific properties attributed to $p=p\left(x_{T}\right)$ are as follows:-

$$
\begin{array}{cc}
p(x)>0 & \text { for all } x \geq 0, \\
D p(x)<0 & \text { for all } x>0, \\
D^{2} p(x)>0 & \text { for all } x>0,
\end{array}
$$

where $D$, without any subscripts, denotes ordinary differentiation and $D^{2}$ second order differentiation. These properties assert that the demand function is a positive, decreasing and concave function respectively.

The total cost incurred by competitor $i$ is given by the function $C_{i}=C_{i}\left(x_{i}\right)$. Hence we are assuming that a competitor's costs depend solely on his own output. In the following analysis, only the marginal costs and the rate of change of these marginal costs will appear. Hence the fixed costs of a competitor may be neglected and our cost function may be considered as the total variable cost. This (variable) cost function is assumed to be everywhere non-negative and marginal costs are a non-increasing function of output; that is

$$
C_{i}\left(x_{i}\right) \geq 0 \text { for all } x_{i} \geq 0,
$$

and

$$
D^{2} C_{i}\left(x_{i}\right) \leq 0 \text { for all } x_{i} \geq 0 .
$$

The following two assumptions will also be used in the subsequent analysis. These are:-

$$
D p\left(x_{T}\right)-D^{2} C_{i}\left(x_{i}\right)<0
$$

and

$$
x_{i} D^{2} p\left(x_{T}\right)+D p\left(x_{T}\right)<0 .
$$

Assumption (2) states that a competitor's marginal costs cannot decline as fast as price. Assumption (3) asserts that the marginal revenue of any competitor is a decreasing function of the total output of his competitors.

The profit function of competitor $i, \pi_{i}$, is given by

$$
\pi_{i}=x_{i} p\left(x_{T}\right)-C_{i}\left(x_{i}\right) \text {. }
$$


The marginal profit function is given by

$$
D_{i} \pi_{i}\left(x_{1}, x_{2}\right)=x_{i} D p\left(x_{T}\right)+p\left(x_{T}\right)-D C_{i}\left(x_{i}\right) ; \quad i=1,2,
$$

where $D_{k} \pi_{i}$ denotes the partial derivative of $\pi_{i}$ with respect to the $k$ th variable. Assumptions (2) and (3) are sufficient to ensure that a competitor's marginal profit is a decreasing function of his own output. We see this by calculating the rate of change of a competitor's marginal profit with respect to his own output. This gives

$$
\begin{aligned}
D_{i, i} \pi_{i}\left(x_{1}, x_{2}\right) & =x_{i} D^{2} p\left(x_{T}\right)+2 D p\left(x_{T}\right)-D^{2} C_{i}\left(x_{i}\right) \\
& =\left\{x_{i} D^{2} p\left(x_{T}\right)+D p\left(x_{T}\right)\right\}+\left\{D p\left(x_{T}\right)-D^{2} C_{i}\left(x_{i}\right)\right\},
\end{aligned}
$$

where $D_{k, l} \pi_{i}$ denotes the partial derivative of $D_{k} \pi_{i}$ with respect to the $l$ th variable. The terms within the braces are each negative by assumptions (2) and (3). Hence we have

$$
D_{i, i} \pi_{i}\left(x_{1}, x_{2}\right)<0 .
$$

The profit function of competitor $i, \pi_{i}$, is thus a convex function of the competitor's own output, $x_{i}$.

\section{Strategies}

The warfare strategy is such that an oligopolist attempts to maximise the difference between his own profit and a weighted sum of his rival's profits. Competitor $i$ thus aims to maximise

$$
\pi_{i}-\frac{1}{n-1} \sum_{j \neq i} a_{i j} \pi_{j}
$$

In the case of a duopoly, this reduces to competitor $i$ attempting to maximise

$$
\pi_{i}-a_{i} \pi_{j}, \quad i, j=1,2 ; i \neq j .
$$

The scalar $a_{i}$ is an indication of the aggressiveness or otherwise of competitor $i$. If $a_{i}<0$, competitor $i$ is attempting to maximise a weighted sum of his own and his rival's profits. When $a_{i}=0$, competitor $i$ is attempting to maximize his own profit, disregarding any improvement or otherwise in his rival's profit. If $a_{i}>0$, competitor $i$ is adopting an antagonistic behaviour of attempting to maximise a weighted difference of his own and his rival's profits. If each duopolist behaves in this way, that is, $a_{i}>0, i=1,2$, we may anticipate that unstable equilibria occur. Stable equilibria may still occur however, as positive profits may be achieved when $a_{i}>0, i=1,2$. However, if $a_{1} a_{2}>1$, it is easily shown that the expressions given by (4) cannot both be positive. 
A choice of $a_{i}<-1$ would be irrational and self-defeating. We thus assume that

$$
a_{i} \geq-1, \quad i=1,2 .
$$

We now restrict ourselves to the duopoly problem of maximising (4). Competitor $i$ thus calculates

$$
D_{i}\left(\pi_{i}-a_{i} \pi_{j}\right)=0
$$

given

$$
D_{i, i}\left(\pi_{i}-a_{i} \pi_{j}\right)<0
$$

Performing these calculations, we find

$$
\left(x_{i}-a_{i} x_{j}\right) D p\left(x_{1}+x_{2}\right)+p\left(x_{1}+x_{2}\right)-D C_{i}\left(x_{i}\right)=0,
$$

with

$$
\left(x_{i}-a_{i} x_{j}\right) D^{2} p\left(x_{1}+x_{2}\right)+2 D p\left(x_{1}+x_{2}\right)-D^{2} C_{i}\left(x_{i}\right)<0 .
$$

We now assume that there is a unique solution $x_{i}^{*}=x_{i}^{*}\left(x_{j}, a_{i}\right)$ maximising (4) and hence satisfying (7) and (8). Implicitly differentiating (7) we find

$$
D_{1} x_{i}^{*}\left(x_{j}, a_{i}\right)=-\frac{\left\{\left(x_{i}^{*}-a_{i} x_{j}\right) D^{2} p\left(x_{i}^{*}+x_{j}\right)+\left(1-a_{i}\right) D p\left(x_{i}^{*}+x_{j}\right)\right\}}{\left\{\left(x_{i}^{*}-a_{i} x_{j}\right) D^{2} p\left(x_{i}^{*}+x_{j}\right)+2 D p\left(x_{i}^{*}+x_{j}\right)-D^{2} C_{i}\left(x_{i}^{*}\right)\right\}},
$$

or

$$
D_{1} x_{i}^{*}\left(x_{j}, a_{i}\right)=\frac{a_{i}\left\{x_{j} D^{2} p\left(x_{i}^{*}+x_{j}\right)+D p\left(x_{i}^{*}+x_{j}\right)\right\}-\left\{x_{i}^{*} D^{2} p\left(x_{i}^{*}+x_{j}\right)+D p\left(x_{i}^{*}+x_{j}\right)\right\}}{\left\{\left(x_{i}^{*}-a_{i} x_{j}\right) D^{2} p\left(x_{i}^{*}+x_{j}\right)+2 D p\left(x_{i}^{*}+x_{j}\right)-D^{2} C_{i}\left(x_{i}^{*}\right)\right\}} .
$$

From (7) it can be seen that when both duopolists have the same marginal costs and are both joint profit maximisers $\left(D C_{1}=D C_{2}\right.$ and $\left.a_{1}=a_{2}=-1\right)$ then $x_{1}^{*}$ and $x_{2}^{*}$ have identical function forms. Hence such a perfectly symmetrical system possesses a symmetrical collusive solution. Each duopolist may be content with or resign themselves to this equal market share situation. Alternatively, one or both might desire a larger market share and increase their aggressiveness towards the rival to try and achieve this aim.

It is unlikely that each duopolist has identical marginal costs or that they exhibit identical levels of aggression. In this case, the expression (9) needs to be examined. The denominator of (9) is just the expression in (8) which is negative. Each term in the numerator is negative by assumption (3). Hence whenever the aggression coefficient $a_{i}$ is negative, the slope of the reaction curve $x_{i}^{*}$ is always negative. This reflects the co-operative behaviour corresponding to $a_{i}<0$. When the competitor $j$ increases his output, $x_{j}$, competitor $i$ decreases his output in order to maximise the joint profit $\pi_{i}-a_{i} \pi_{j}$.

When $a_{i}=0$, competitor $i$ is attempting to maximise his own profit, $\pi_{i}$. In this case, $x_{i}^{*}=x_{i}^{*}\left(x_{j}\right)$ is just the familiar Cournot reaction curve for competitor $i$. Again this curve always has a negative slope. 
The properties of the reaction schedule are more complicated, and more interesting, for the case of $a_{i}>0$. The qualitative behaviour can most easily be seen for the rivalistic case of $a_{i}=1$. In this case, $D_{1} x_{i}^{*}$ has the same sign as $x_{i}^{*}-x_{j}$. Then the assumed properties of the market functions are such that if $x_{i}^{*}>x_{j}$, then $0<D_{1} x_{i}^{*}<1$ and if $x_{i}^{*}<x_{j}$, then $-1<D_{1} x_{i}^{*}<0$. If $x_{i}^{*}=x_{j}$, then $D_{1} x_{i}^{*}=0$.

The warfare strategy for a rivalistic firm depends on the relative market shares of the two firms. If such an aggressive firm has the greater market share, then its optimal behaviour is to follow increases in its rival's output with increases in its own output but not to match such increases. If the firm has the smaller market share, its optimal strategy is to decrease its output when the rival increases its output, but the decrease is to be less than the rival's increase.

In either case, the strategy is seen as one of attempting to control total market output in such a manner as to place the maximum pressure on the rival. For the firm with the smaller market share, this increase in market output has to be controlled by decreasing its own output. Such output decreases are not usually associated with an aggressive firm. Indeed, from the opposition's view, it will not be obvious which strategy the firm is adopting. Along a co-operative firm's reaction schedule $\left(a_{i}=-1\right)$, total market output is always decreasing. That is, increases in one firm's output are more than compensated by decreases in the co-operative firm's output. It is thus obvious to the rival that a firm is adopting a co-operative approach.

However, for any Cournot firm $\left(a_{i}=0\right)$ or a somewhat aggressive firm $\left(a_{i}>0\right)$ with the smaller market share, decreases in its output do not match the other firm's increases. This can also occur for a firm that is mildly co-operative; that is a firm with an aggression coefficient that is negative but near zero. Hence the other firm cannot be confident it knows the level of aggression being adopted by its rival.

\section{Stability analysis}

In this section we present the stability analysis of the duopoly system, with both competitors using the strategies outlined above.

Given competitor $j$ 's output $x_{j}(t)$, the output $x_{i}^{*}\left(x_{j}(t), a_{i}\right)$ is the output competitor $i$ would like to produce. However, in practice, a large change in output from $x_{i}(t)$ to $x_{i}^{*}(t)$ may not be possible. By comparing $x_{i}(t)$ to $x_{i}^{*}(t)$, competitor $i$ will see in which direction his output needs to change. With this in mind, we use the adjustment process employed by Fisher, namely

$$
D x_{i}(t)=k_{i}\left(x_{i}^{*}-x_{i}\right), \quad i=1,2,
$$

where $D x_{i}(t)$ denotes differentiation with respect to time. 
The coefficients $k_{i}$ are positive scalars. The adjustment process is thus designed so that firm $i$ is always adjusting its output towards $x_{i}^{*}(t)$.

The constant $k_{i}$ may be interpreted as a measure of the "speed of adjustment" of firm $i$. Alternatively, it may be thought of as a "confidence factor", indicating the degree of confidence firm $i$ has in its theoretical result $x_{i}^{*}(t)$. Hence $k_{i}=1$ would indicate full confidence in the calculated figure.

Equilibrium points of $(10)$ are points

$$
x_{1}^{*}\left(\beta_{i}\right)=\alpha_{i}, \quad \text { and } x_{2}^{*}\left(\alpha_{i}\right)=\beta_{i} .
$$

We will assume that the two curves $x_{1}^{*}$ and $x_{2}^{*}$ intersect at a finite number of points $\left(\alpha_{i}, \beta_{i}\right)$ and include the possibility of no intersection point at all. This latter case is interesting in that it leads to one of the duopolists producing zero output and the successful duopolist, say competitor $i$, producing his monopoly output of $x_{i}^{*}(0)$. Which of the two duopolists is forced to a zero output is determined by the relative positions of the two reaction curves. This positioning depends in turn on the particular demand and cost curves pertaining to the duopoly and the aggressiveness factors $a_{i}$.

The equilibrium points $\left(\alpha_{i}, \beta_{i}\right)$ will be a series of consecutive stable and unstable points. This is easily seen by considering the sign of $D x_{1}$ and $D x_{2}$ along the reaction curves $x_{1}^{*}$ and $x_{2}^{*}$. Clearly then the equilibrium points can only be locally stable or unstable. This precludes an analysis based on global techniques, such as that in Seade [6]. The analysis of local stability properties presented below may be found in a text such as Hirsch and Smale [5].

To investigate the stability of the system at equilibrium points, we linearise the differential equation (10) about the point $e_{i}=\left(\alpha_{i}, \beta_{i}\right)$.

If we introduce the change of variables, $u=x_{1}-\alpha_{i}$ and $v=x_{2}-\beta_{i}$, then the system of equations (10) may be written

$$
\left[\begin{array}{l}
D U(t) \\
D V(t)
\end{array}\right]=\left[\begin{array}{cc}
-k_{1} & k_{1} D x_{1}^{*}\left(\beta_{i}\right) \\
k_{2} D x_{2}^{*}\left(\alpha_{i}\right) & -k_{2}
\end{array}\right]\left[\begin{array}{l}
U \\
V
\end{array}\right]+\text { non-linear terms. }
$$

The eigenvalues of $D f\left(e_{1}\right)$ are thus

$$
\lambda_{ \pm}=-\frac{1}{2}\left(k_{1}+k_{2}\right) \pm \frac{1}{2}\left\{\left(k_{1}-k_{2}\right)^{2}+4 k_{1} k_{2} q_{i}\right\}^{1 / 2},
$$

where, for notational convenience, we have introduced the term

$$
q_{i}=D x_{1}^{*}\left(\beta_{i}\right) \cdot D x_{2}^{*}\left(\alpha_{i}\right) .
$$

If $\left(k_{1}-k_{2}\right)^{2}+4 k_{1} k_{2} q_{i} \leq 0$, the equilibrium point $\left(\alpha_{i}, \beta_{i}\right)$ is asymptotically stable, as the eigenvalues are complex with negative real parts.

For unstable equilibria to occur, it is necessary that $\left(k_{1}-k_{2}\right)^{2}+4 k_{1} k_{2} q_{i}>0$. In this case $\lambda_{+}$and $\lambda_{-}$are real, distinct eigenvalues. 
By calculating the magnitudes of the two eigenvalues, it is easily seen that for stability to occur, it is necessary that $q_{i} \leq 1$ and sufficient that $q_{i}<1$.

The question arises as to what effect the aggression coefficients, $a_{1}$ and $a_{2}$, have on the stability of an equilibrium point $\left(\alpha_{i}, \beta_{i}\right)$. We have developed the necessary conditions and sufficient conditions for such a point to be stable, in the form $q_{i} \leq 1$ and $q_{i}<1$ respectively, where $q_{i}=D_{1} x_{1}^{*}\left(\beta_{i}, a_{1}\right) D_{1} x_{2}^{*}\left(\alpha_{i}, a_{2}\right)$. From (9) we have $D_{1} x_{1}^{*}\left(\beta_{i}, a_{1}\right)$ and $D_{1} x_{2}^{*}\left(\alpha_{i}, a_{2}\right)$ as functions of $\alpha_{i}, \beta_{i}$ and $a_{1}$ or $a_{2}$ respectively. Thus we can write the inequality $q_{i}<1$ as

$$
q_{i}=G\left(\alpha_{i}, \beta_{i}, a_{1}\right) \cdot H\left(\alpha_{i}, \beta_{i}, a_{2}\right)<1,
$$

where $G\left(\alpha_{i}, \beta_{i}, a_{1}\right)=D_{1} x_{1}^{*}\left(\beta_{i}, a_{1}\right)$ and $H\left(\alpha_{i}, \beta_{i}, a_{2}\right)=D_{1} x_{2}^{*}\left(\alpha_{i}, a_{2}\right)$. But $\alpha_{i}$ and $\beta_{i}$ are both functions of $a_{1}$ and $a_{2}$. Thus $q_{i}=q_{i}\left(a_{1}, a_{2}\right)$. How $q_{i}$ varies as either $a_{1}$ or $a_{2}$ is varied is rather complicated, due to a change in $a_{i}$ causing a change in both $\alpha_{i}$ and $\beta_{i}$.

The convexity of the curves $x_{1}^{*}$ and $x_{2}^{*}$ at the point $\left(\alpha_{i}, \beta_{i}\right)$ as well as the slopes of these two curves at the point $\left(\alpha_{i}, \beta_{i}\right)$ plays a part in determining the effect on $q_{i}$ of a change in $a_{1}$. From this it is obvious that the effect is difficult to describe. However some partial results may be obtained by using the expressions (9) evaluated at $\left(\alpha_{i}, \beta_{i}\right)$ for the functions $G$ and $H$. The inequality $q_{i}<1$ then becomes

$$
\begin{aligned}
\left(a_{1} a_{2}\right. & -1)\left(\alpha_{i}+\beta_{i}\right) D p\left(\alpha_{i}+\beta_{i}\right) D^{2} p\left(\alpha_{i}+\beta_{i}\right) \\
& +\left[\left(a_{1}-1\right)\left(a_{2}-1\right)-4\right]\left\{D p\left(\alpha_{i}+\beta_{i}\right)\right\}^{2} \\
< & -D^{2} C_{1}\left(\alpha_{i}\right)\left\{\left(\beta_{i}-a_{2} \alpha_{i}\right) D^{2} p\left(\alpha_{i}+\beta_{i}\right)+2 D p\left(\alpha_{i}+\beta_{i}\right)-D^{2} C_{2}\left(\beta_{i}\right)\right\} \\
& -D^{2} C_{2}\left(\beta_{i}\right)\left\{\left(\alpha_{i}-a_{1} \beta_{i}\right) D^{2} p\left(\alpha_{i}+\beta_{i}\right)+2 D p\left(\alpha_{i}+\beta_{i}\right)-D^{2} C_{1}\left(\alpha_{i}\right)\right\} \\
& -D^{2} C_{1}\left(\alpha_{i}\right) \cdot D^{2} C_{2}\left(\beta_{i}\right) .
\end{aligned}
$$

If both competitors have constant marginal costs, inequality (14) may be reduced to

$$
\left(a_{1} a_{2}-1\right)\left(\alpha_{i}+\beta_{i}\right) \cdot D^{2} p\left(\alpha_{i}+\beta_{i}\right)+\left[\left(a_{1}-1\right)\left(a_{2}-1\right)-4\right] \cdot D p\left(\alpha_{i}+\beta_{i}\right)>0 .
$$

This in turn may be expressed in the form

$$
\left(a_{1} a_{2}-1\right)\left[\left(\alpha_{i}+\beta_{i}\right) D^{2} p\left(\alpha_{i}+\beta_{i}\right)+2 D p\left(\alpha_{i}+\beta_{i}\right)\right]-\left(a_{1}+1\right)\left(a_{2}+1\right) D p\left(a_{i}+\beta_{i}\right)>0 \text {. }
$$

By assumption (3) we have $\alpha_{i} D^{2} p\left(\alpha_{i}+\beta_{i}\right)+D p\left(\alpha_{i}+\beta_{i}\right)<0$ and $\beta_{i} D^{2} p\left(\alpha_{i}+\beta_{i}\right)+D p\left(\alpha_{i}+\beta_{i}\right)<0$. From assumption (1b) we have $-D p\left(\alpha_{i}+\beta_{i}\right)>0$ and assumption (5) implies that $\left(1+a_{1}\right)\left(1+a_{2}\right) \geq 0$. Hence inequality (16) will be satisfied when $a_{1} a_{2}-1 \leq 0$ but not both $a_{1}$ and $a_{2}$ are equal to -1 . If $a_{1}=a_{2}=-1$, then (16) is identically zero. The case of $a_{1}=a_{2}=-1$ and constant marginal costs needs to be considered separately. Referring back to (9), we see that if $D^{2} C_{i} \equiv 0$, then $D_{1} x_{i}^{*}\left(x_{j},-1\right)=-1$. Hence 
the curves $x_{1}^{*}$ and $x_{2}^{*}$ are parallel. From (7), we observe that if the marginal costs of the duopolists are the same, then $x_{1}^{*}$ and $x_{2}^{*}$ are coincident lines. If the marginal costs are different, then the curves $x_{1}^{*}$ and $x_{2}^{*}$ are distinct parallel lines. Thus we have either a continuum of intersection points of the curves $x_{1}^{*}$ and $x_{2}^{*}$ or no intersection points. In the first case, as Bishop pointed out, we cannot predict the outcome. If firm $i$ maintains its co-operative strategy of $a_{i}=-1$ and its rival increases its output, then firm $i$ must lower its own output by the corresponding increase of its rival's output. In the second case of no intersection point, all trajectories tend to one of the monopoly points $x_{1}^{*}(0)$ or $x_{2}^{*}(0)$. In actual fact, the trajectories tend to the monopoly point with the lowest marginal cost. Clearly, it is self-destructive for the firm with the highest marginal costs, finding itself in the above situation, to be so co-operative. By doing so, it is obligingly eliminating itself from the market.

If both competitors have decreasing marginal costs, inequalities (15) or (16) are necessary conditions for stability. In particular, if $a_{1}=a_{2}=-1$, we see that inequality (14) is not satisfied. Further, from (9), it readily follows that $q_{i}(-1,-1)>1$. Then all equilibrium points corresponding to joint-maximisation strategies (that is $a_{1}=a_{2}=-1$ ) are unstable. As there are no closed trajectories anywhere in the phase-plane, the trajectories must tend to one of the monopoly outputs.

We found above that $q_{i} \leq 0$ is a sufficient condition for stability. For $q_{i}<0$, the numerators of $D_{1} x_{1}^{*}\left(\beta_{i}, a_{1}\right)$ and $D_{1} x_{2}^{*}\left(\alpha_{i}, a_{2}\right)$ must have opposite signs. If we let

$$
A=\alpha_{i} D^{2} p\left(\alpha_{i}+\beta_{i}\right)+D p\left(\alpha_{i}+\beta_{i}\right)
$$

and

$$
B=\beta_{i} D^{2} p\left(\alpha_{i}+\beta_{i}\right)+D p\left(\alpha_{i}+\beta_{i}\right),
$$

then we see, from (9), that $q_{i} \leq 0$ is equivalent to

$$
\left[a_{1} B-A\right] \cdot\left[a_{2} A-B\right] \leq 0
$$

or

$$
-a_{1} B^{2}-a_{2} A^{2}+\left(1+a_{1} a_{2}\right) A B \leq 0 .
$$

From the theory of quadratic forms, we can deduce that $q_{i} \leq 0$ is satisfied for $a_{1}, a_{2} \geq 0, a_{1} a_{2}=1$. In particular, the rivalistic case of $a_{1}=a_{2}=1$ is always stable. This analysis for $q_{i} \leq 0$ is valid regardless of whether the marginal costs are decreasing, constant or increasing-the rivalistic case is stable in all cases.

\section{Conclusion}

The analysis has been presented of a duopoly model which incorporates a degree of flexibility in behaviour of the firms. This flexibility is such that a 
firm can vary its strategy from co-operation through to antagonism towards the rival. One obvious conclusion is that if both competitors myopically attempt to maximise their joint profit by adjusting their own output, the resulting system is unstable and will in general lead to the elimination of one firm from the market. Clearly it is not in the interest of this firm to persist with this strategy.

All equilibrium points are stable for the rivalistic strategies of $a_{1}=a_{2}=1$. This result holds regardless of the cost functions, that is, regardless of whether the marginal costs are increasing, decreasing or constant.

This persistence with antagonistic behaviour could be caused by a number of factors. For instance, one of the duopolists may believe that, by attacking his rival strongly enough, thereby causing both competitors to suffer a loss, he may eventually drive the rival from the market. Another reason for the persistent aggressive behaviour may be due to an element of bluffing. The suspicion that his rival's aggressive behaviour is a bluff may induce a competitor to maintain his own aggressive stance, hoping eventually to call his opponent's bluff. The model does not include any mechanism for a competitor to withdraw from such a long-term stalemate. In practice, he may not be able to sustain this stalemate position indefinitely if this entails a protracted loss.

From the stability analysis we found that a sufficient condition for stability is $q_{i}<1$ and a necessary condition for stability is $q_{i} \leq 1$.

The algebraic expressions $q_{i}<1$ have a geometric interpretation concerning the manner in which the two reaction curves $x_{1}^{*}$ and $x_{2}^{*}$ intersect at an equilibrium point. It will always be satisfied if one of $x_{1}^{*}$ and $x_{2}^{*}$ is increasing and the other is decreasing. While it was not possible to give definitive economic criteria for $q_{i}<1$, some interesting results were found.

\section{References}

[1] R. L. Bishop, "Duopoly: Collusion or Warfare", Amer. Econ. Rev., 50 (1960) 933-961.

[2] A. Cournot, Researches into the Mathematical Principles of the Theory of Wealth, (trans. N. T. Bacon, N.Y., 1929).

[3] J. W. Friedman, Oligopoly and the Theory of Games, (North-Holland, Amsterdam, 1977).

[4] N. J. Hathaway, I. W. Murray, and J. A. Rickard, "The Stability of Bishop's Warfare Strategy", J. Austral. Math. Soc. Ser. B, 21 (1980) 418-432.

[5] M. W. Hirsch, and S. Smale, Differential Equations, Dynamical Systems and Linear Algebra, (Academic Press, N.Y., 1974).

[6] J. Seade, "The Stability of Cournot Revisited", J. Econom. Theory, 23 (1980) 15-27. 